\title{
Constructing a SMBL-based S-system Simulation Platform
}

\author{
Jie-Liang Luo \\ Department of Computer Science and Information Engineering, National Taiwan University \\ Email: p93922004@ntu.edu.tw \\ D. Frank Hsu \\ Department of Computer Science and Information Engineering, Fordham University \\ Email: hsu@cis.fordham.edu \\ Cheng-Yen Kao \\ Department of Computer Science and Information Engineering, National Taiwan University \\ Email: cykao@csie.ntu.edu.tw
}

\section{Introduction}

XML (eXtensible Markup Language) is a commonly used markup language for electronic data exchange. XML has been extended to systems biology. SBML (systems biology markup language) [4] is developed by ERATO Kitano project, California Institute of Technology, USA. SBML take XML and UML modeling as common element to describe and analyze systems biology, and it can be used for biologists for exchanging biological pathway data among different systems biology modeling and simulation software.

There are many existing tools can manipulate SBML formats, e.g. KEGG2SBML [1], CellDesigner, and MathSBML [2]. The combination of these software tools facilitates the analysis of the mathematical modeling for biological reactions. However, it is still an open issue to develop a integrated platform to provide the transformation of data formats, editing, simulation, and analysis in real time. In this study, we design an integrated web service to fetch biological pathway data, convert to visual graphically interface, transform to mathematical modeling, and solve the model in real time.

\section{Methods}

Systems biology is an approach to analyze the biological chemical reactions and its corresponding mathematic modeling. Also applying mathematical modeling to simulate and solve the equations for understanding the mechanism of biological chemical reactions. The mathematical modeling simulation also can be used as evaluation and prediction of biological systems. Therefore, the strategy of the proposed platform can be completed with the following steps:

Step1 list all elements affected by systems

0-7803-9329-5/05/\$20.00 C2005 IEEE
Step2 list all reactions affected by these elements

Step3 transform these elements and reactions into graphs

Step4 transform the graph into S-system model [3]

Step5 solve and analyze S-system model

Step6 explain the mechanism of biological reactions.

\section{Implementation}

We design a platform to provide the functionality of SBML editing and S-system simulation to help users to analyze and solve the mathematical model. The following Figure shows the architecture of our proposed platform system.

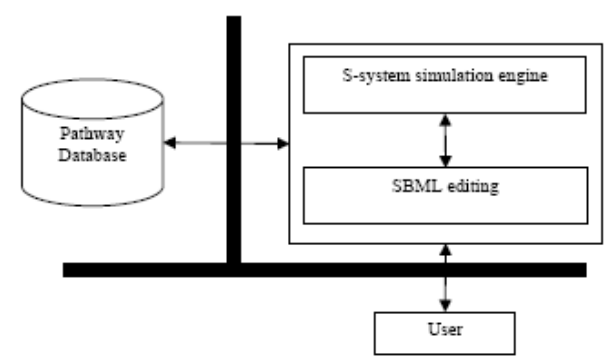

The platform contains the following functionalities:

1. SMBL editing: provides a user friendly interface to construct biological chemical reactions as SBML formats. Also each parameter of each element and reaction can be modified by user.

2. S-system simulation engine: transforms SBML file formats into S-system mathematical models. Also, the models be solved by the mathematical equations, and the results of simulation dynamically can be 
displayed.

\section{References}

[1] Akira Funahashi, Akiya Jouraku, Hiroaki Kitano. 2004. Converting the KEGG Pathway Database to SBML. ICSB2004, Heidelberg, Germany, October 9-13.

[2] B. Shapiro, M. Hucka, A. Finney and J. C. Doyle. 2004. MathSBML: A Package for Manipulating SBML-Based Biological Models. Bioinformatics, 20(16): 2829-2831.

[3] E. O. Voit. Computational Analysis of Biochemical Systems. A Pratical Guide for Biochemists and Molecular Biologists. Cambridge University Press, 2000

[4] M. Hucka, A. Finney, B.J. Bornstein, S.M. Keating, B.E. Shapiro, J. Matthews, B.L. Kovitz, M.J. Schilstra, A. Funahashi, J.C. Doyle, H. Kitano. 2004. Evolving a Lingua Franca and Associated Software Infrastructure for Computational Systems Biology: The Systems Biology Markup Language (SBML) Project. Systems Biology June, 1(1): 41-53. 